\title{
8. Firing Wave Instability and Chaos of Current Filaments in Semiconductor
}

\section{$\operatorname{AUTHOR}(\mathrm{S})$ :}

AOKI, Kazunori; YAMAMOTO, Keiichi; MUGIBAYASHI, Nobumichi

\section{CITATION:}

AOKI, Kazunori ... [et al]. 8. Firing Wave Instability and Chaos of Current Filaments in Semiconductor. 物性研究 1988, 50(4): 543-544

ISSUE DATE:

1988-07-20

URL:

http://hdl.handle.net/2433/93156

RIGHT: 
8. Firing Wave Instability and Chaos of Current Filaments in Semiconductor

Kazunori AOKI, Keiichi YAMAMOTO and Nobumichi MUGIBAYASHI

Department of Electrical and Electronic Engineering, Faculty of Engineering, Kobe University, Rokkodai, Nada, Kobe 657

Firing wave instability and chaos of current filaments have been investigated in high-purity $\mathrm{n}$-GaAs at $4.2 \mathrm{~K}$. Under the pulse voltage of $38 \mathrm{kHz}$ and with the weak photoexcitation, the current filaments formed by impact ionization of the neutral shallow donors exhibit the firing wave instability. The macro-ensemble of the firing density wave (coarsegrained signals) detected by a lock-in amplifier shows various types of periodic oscillations and chaos (including period doubling bifurcation) at extremely low frequencies less than a few $\mathrm{Hz}$ ! The oscillatory behaviors of the firing density wave have been presented as a novel phenomenon in a sense that it can be uniquely explained by the "coarsegrained picture" 1-3) which has been treated in the neural network theory ${ }^{4,5)}$. The important aspects obtained in the experiments are as follows;

(a) The oscillatory behaviors are caused by the mutual interaction between or among multi-current filaments.

(b) An analogy with the neural network theory is satisfactory.

(c) The correlation dimension of the chaotic motion supports the deterministic nature in the firing wave instability.

Besides the period doubling bifurcation, we often observe the intermittent behaviors which are very similar to the turbulence in fluid system observed by Ahlers and Walden ${ }^{6)}$. An example is shown in Fig.l. As shown in Fig.lb, there are two phases, where each phase is trapped stably in a minimum potential but is adjacent to another minimum potential with a certain activation energy ${ }^{6}$. With the small external noise embedded in the system, the intermittent behavior occurs due to the random jumps between two phases. 
(a) $3.45 \mathrm{~V} / \mathrm{cm}$

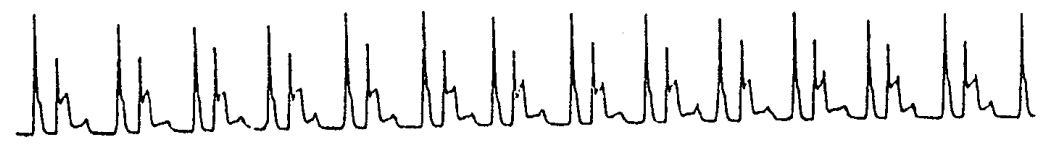

(b) $3.64 \mathrm{~V} / \mathrm{cm}$

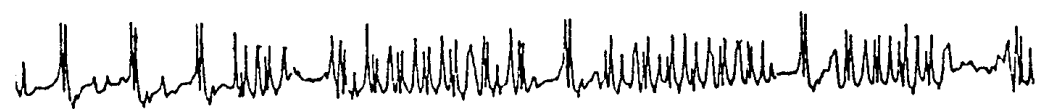

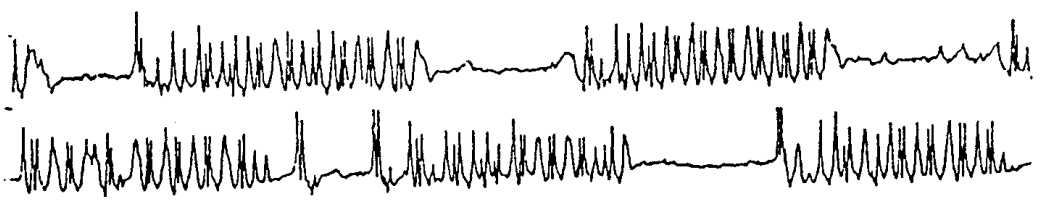

(c) $3.65 \mathrm{~V} / \mathrm{cm}$

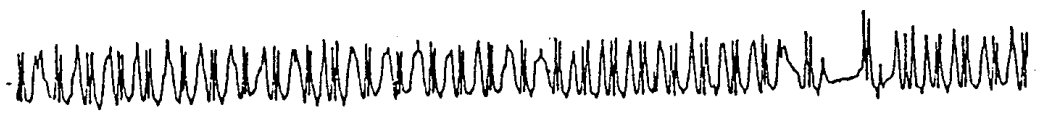

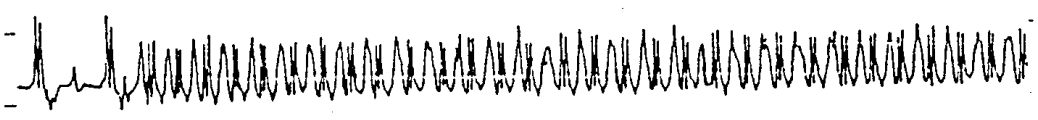

(d) $3.68 \mathrm{v} / \mathrm{cm}$

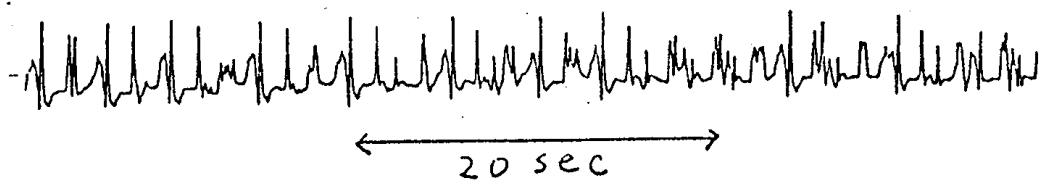

Fig. 1 An example of intermittency observed as a function of the electric field. The electric field strength is simply evaluated by the pulse height devided by the spacing between the contacts.

1) K. Aoki, T. Kobayashi and K. Yamamoto: J. Phys. Soc. Jpn. 51 (1982) 2373.

2) K. Aoki and K. Yamamoto: Phys. Lett. 98A (1983) 72.

3) K. Aoki, O. Ikezawa and K. Yamamoto: Phys. Lett. 98A (1983) 217;

K. Aoki, O. Ikezawa, N. Mugibayashi and K. Yamamoto: Physica 134B (1985) 288.

4) S. Amari: Kybernetik 14 (1974) 201.

5) S. Amari: IEEE Trans. Syst. Man Cybern. SMC-2 (1972) 643.

6) G. Ahlers and R.W. Walden: Phys. Rev. Lett. 44 (1980) 445. 